\title{
BMJ Open Inequalities in family practitioner use by sexual orientation: evidence from the English General Practice Patient Survey
}

\author{
Sean Urwin, William Whittaker
}

To cite: Urwin S,

Whittaker W. Inequalities in family practitioner use by sexual orientation: evidence from the English General Practice Patient Survey. BMJ Open 2016;6:e011633. doi:10.1136/bmjopen-2016011633

- Prepublication history and additional material is available. To view please visit the journal (http://dx.doi.org/ 10.1136/bmjopen-2016011633).

Received 22 February 2016 Revised 29 March 2016 Accepted 11 April 2016

CrossMark

Manchester Centre for Health Economics, University of Manchester, Manchester, UK

Correspondence to

Sean Urwin;

sean.urwin@manchester.ac.uk

\section{ABSTRACT}

Objective: To test for differences in primary care family practitioner usage by sexual orientation.

Design: Multivariate logistic analysis of pooled crosssectional postal questionnaire responses to family practitioner usage.

Setting: Patient-reported use and experience of primary care in England, UK. Data from several waves of a postal questionnaire (General Practice Patient Survey) 2012-2014.

Population: 2807320 survey responses of adults aged 18 years and over, registered with a family practitioner.

Main outcome measures: Probability of a visit to a family practitioner within the past 3 months.

Results: Lesbian women were 0.803 times $(95 \% \mathrm{Cl}$ 0.755 to 0.854 ) less likely to have seen a family practitioner in the past 3 months relative to heterosexual women (bisexual women $\mathrm{OR}=0.887,95 \%$ $\mathrm{Cl} 0.817$ to 0.963$)$. Gay men were 1.218 times (95\% $\mathrm{Cl} 1.163$ to 1.276 ) more likely to have seen a family practitioner relative to heterosexual men (bisexual men $\mathrm{OR}=1.084,95 \% \mathrm{Cl} 0.989$ to 1.188 ). Our results are robust to the timing of the family practitioner visit $(0-3,0-6,0-12$ months). Gay men were more likely to have seen a family practitioner than heterosexual men where the proportion of women practitioners in the practice was higher $(\mathrm{OR}=1.238,95 \% \mathrm{Cl} 1.041$ to 1.472).

Conclusions: Inequalities in the use of primary care across sexual orientation in England exist having conditioned on several measures of health status, demographic and family practitioner characteristics. The findings suggest these differences may be reduced by policies targeting a reduction of differences in patient acceptability of primary care. In particular, further research is needed to understand whether lower use among heterosexual men represents unmet need or overutilisation among gay men, and the barriers to practitioner use seemingly occurring due to the gender distribution of practices.

\section{INTRODUCTION}

The family practitioner plays a key role in most healthcare systems worldwide. In addition to being the first port of call for

\section{Strengths and limitations of this study}

- The study uses a large-scale population-based sample allowing us to adjust for a rich set of factors that may bias the influence of sexual orientation on access to primary care.

- The ability to match family practitioner workforce characteristics in the data permits an analysis of the role of supply-side factors on access to primary care.

- Our main outcome measures whether a visit occurred within a time period but not the content and volume of family practitioner visits.

- Health measures are self-reported and may not be clinically diagnosed.

- Differences in institutional settings and tolerance towards sexual minority individuals may limit the generalisability of results.

primary care, they are typically the gatekeepers for secondary care. Effective and equitable access to healthcare, therefore, relies on a good patient-practitioner relationship. Many countries with publicly provided healthcare aim to provide access to healthcare on the basis of the need for care, with many stating that access should not depend on sexual orientation. ${ }^{12}$ However, evidence of inequalities in health according to sexual orientation across the world exists, ${ }^{3-8}$ calling for an understanding into the factors that result in sexual orientation-specific health inequalities. Avoidable inequalities in health may arise due to variation in access to healthcare. Access to healthcare is a multifaceted concept, dependent not only on need, but on the availability of healthcare (supply-side provision factors), patient acceptability for care, and the affordability of healthcare. ${ }^{9} 10$ Differences in access to healthcare by sexual orientation groups due to the need for, availability of, or affordability of healthcare would not suggest sexual orientation-specific inequity of access. Sexual orientation-specific inequity arises where discriminatory expectations or misconceptions between patient and 
provider causes issues of patient acceptability (acceptance of seeking care) and may result in reduced use of healthcare among minority sexual orientation groups. There is evidence to suggest that patient acceptability of care may be an issue, as recent reports have highlighted that discriminatory attitudes of providers of healthcare are felt by minority sexual orientation groups. ${ }^{11-14}$

While there is an extensive literature on inequalities in healthcare by ethnic group membership and social class, ${ }^{15} 16$ little is known about inequalities by sexual orientation. As such, the evidence required to support the need for policies to address inequities in access to family practitioners by sexual orientation has been limited. The lack of evidence of inequalities in England has resulted in, for example, no specific sexual orientation inequalities adjustment factor being applied to healthcare budget allocations. ${ }^{17}$ This contrasts with the range of socioeconomic, ethnicity, age and gender factors that combine to determine healthcare budget shares to local geographic areas in England. ${ }^{18}$

The existing literature is mixed, ${ }^{19}$ but there is some evidence that suggests lesbian, gay and bisexual individuals use their family practitioner in different amounts. Several studies indicate lesbian women have lower family practitioner use than heterosexual women, ${ }^{6}{ }^{20-22}$ while gay men have higher family practitioner usage than their heterosexual counterparts. ${ }^{23-25}$ The literature is largely focused in North America, ${ }^{6}{ }^{20-22} 26$ suffers from poorly representative samples which are not generalisable to a particular country, ${ }^{202127}$ is comprised of small non-heterosexual sample sizes, ${ }^{7} 212728$ and/or includes only one or two of the possible non-heterosexual sexual orientation categories for analysis. ${ }^{6} 7212426-28$ Further, to identify inequalities in access to healthcare attributable to sexual orientation requires access to be conditional on the need, availability and affordability of healthcare. Many studies use poor measures of physical health, mental health and health behaviours ${ }^{21} 222629$ which may confound the sexual orientation effect by need; and not all of them include provider characteristics. $^{6} 7{ }^{21-30}$ Each of these limitations may severely bias estimates of sexual orientation-specific inequity of access to primary care.

In this paper, we aim to identify whether there are inequalities in healthcare use by sexual orientation groups in England. We hypothesise that (1) the unadjusted effects of sexual orientation on use are biased, partly reflecting the need, availability and affordability of healthcare domains, and (2) that after accounting for confounding, significant differences exist in family practitioner use by sexual orientation status. Our analysis is based on General Practitioner Patient Survey (GPPS) data, a large-scale survey of randomly selected individuals registered with a family practitioner in England. A lack of evidence-based sexual orientation policy arises because evidence of inequity is largely compiled based on activity data of healthcare use (used as a proxy for access) with sexual orientation rarely observed.
We seek to address this gap and, in doing so, inform sexual orientation policy by highlighting whether policy should be developed, and to whom it should be directed.

\section{METHODS}

To test whether there are statistically significant differences in family practitioner usage by sexual orientation we estimated multivariate logistic regression models. These included patient-reported family practitioner use regressed against self-reported sexual orientation and a range of potential confounding variables using pooled cross-sectional data from the GPPS.

\section{Data}

Data on family practitioner use was obtained via the GPPS. The GPPS began in 2007 and was intended to provide information on patient experience of their family practitioner practice in England, UK. Annually, approximately 2.6 million surveys are sent to randomly selected patients from each practice list. The GPPS is a postal survey, though completion can be made online or over the telephone. Sample selection is based on practice registration records: an individual must have a valid National Health Service number, be 18 years of age or older, and have been registered with the practice for at least 6 months. ${ }^{31}$ The data are cross-sectional; patients may be surveyed more than once over survey years, but only once within a 12-month period. The sample size is constructed so that $95 \%$ CIs can be obtained for each practice for each question in the survey. The annual response rate is approximately $35 \%$ ( 1 million patients). Half are surveyed January-March, and the other half July-September.

Since 2011, new weights to account for age, gender and deprivation are available. The new weights of the survey mean recent waves of the GPPS are only comparable from June 2011. Weighting is essential to ensure that responses are broadly in line with practice populations. The weights in the GPPS account for unequal probability of selection, non-response (which factors in age, gender, location and deprivation (using the individual's postcode), ethnicity, marital status, overcrowding, household tenure and employment status), and calibration for practice representativeness. The weights provided are used in each model specification and restrict our data to six waves of the survey (2012-2014).

The GPPS data are ideal for an analysis of family practitioner use and sexual orientation due to the sample size. A large sample size of sexual orientation minority groups allows for better identification of statistical associations; whereas small samples create issues of biased inference. ${ }^{32}$ Furthermore, comparisons to larger sexual orientation groups (heterosexuals) is possible. In addition, the range of patient characteristics recorded and the ability to match practice-level characteristics to patients enabled a comprehensive assessment of the domains of access to family practitioners. 


\section{Primary outcome measure}

Respondents are asked 'When did you last see or speak to a GP (family practitioner) from your GP surgery?' (the options are: 'in the past 3 months'; 'between 3 and 6 months'; 'between 6 and 12 months'; 'more than 12 months ago'; or 'never visited the family practitioner'). Our primary outcome measure is a binary indicator for whether the respondent had seen a family practitioner from their practice within the past 3 months; all other responses form the null group. Past 3 months usage was chosen as the primary outcome measure for several reasons. First, recall error may be smaller for more recent attendance. Second, our methodology attempted to account for potential confounding using current patient and practice characteristics that may change over a longer retrospective period.

\section{Statistical analyses}

We estimated multivariate logistic regressions of family practitioner use. Our main explanatory variable of interest was sexual orientation. In the GPPS, sexual orientation is asked based on the respondent's perceived identity: heterosexual, lesbian or gay, bisexual, other and 'prefer not to say'. The latter two options, and a category for those who did not answer, were included because some respondents may not categorise themselves into a particular identity. Models were stratified by gender. Gender stratification was chosen due to the relative physiological differences that may exist between men and women and could result in alternative determinants of family practitioner use. Models were weighted using the GPPS weights for sample representativeness. ${ }^{33}$ To account for potential heteroskedasticity (which may lead to biased inference), robust SEs were used and clustered at the practice level.

\section{Confounding}

Our measure of use as a proxy for access to a family practitioner was problematic, since access to any form of healthcare provider is multidimensional. Access for those with equal need is dependent on how available services are (geographically, queuing); affordability (whether the respondent is employed and/or can take time to seek healthcare); and acceptability (patient and provider expectations). ${ }^{9}$ In order to identify the effects of sexual orientation, it is important to remove any confounding factors that are representative of domains of access that, while correlated with sexual orientation, are not specifically caused by sexual orientation. For example, if age were correlated with family practitioner visits, but also correlated with sexual orientation, then the estimated effect of sexual orientation would partly reflect the effect of younger ages on attendance.

To identify the relationships between sexual orientation and domains of access we first estimated a univariate logistic model of family practitioner use against sexual orientation. We then included patient characteristics, and finally included practice characteristics as well.

\section{Patient characteristics}

A key issue for this study is ensuring that differences in use by sexual orientation are not explained by differential levels of need. Healthcare systems that base access on the need for care make the subjective decision that differences in use by sexual orientation that arise due to differences in need are justified. Numerous studies have shown evidence of significant health disparities by sexual orientation. ${ }^{3-72123242730}$ To ensure that the estimated effects of sexual orientation are not confounded by (potentially justifiable) differences in the need for healthcare, we include age (eight categories: 18-24, 2534, 35-44, 45-54, 55-64, 65-74, 75-84 and 85 years and over), and several self-reported measures of need: the presence of an acute ill health condition (whether the respondents activities have been limited at the date of the survey due to the presence of an illness which lasts a few days or weeks), health-related quality of life (measured via the EQ_5D score ${ }^{\mathrm{i}}$ ), ${ }^{34}$ a medical condition (17 categories $^{\mathrm{ii}}$ ), presence of a long-standing health condition, and smoking status (four categories: never smoked, former smoker, occasional smoker and regular smoker).

To capture characteristics that may signify avoidable inequalities in health we used several additional patient characteristics: ethnicity (six categories: White, Mixed, Black, Asian, Chinese and Other), religious status (nine categories: no religion, Buddhist, Christian, Hindu, Jewish, Muslim, Sikh, other, and 'prefer not to say') and employment status (eight categories: full-time paid work, part-time paid work, full-time education, unemployed, permanently sick or disabled, fully retired from work, looking after the home, and doing something else). The GPPS also contains information on the respondent's local area of residence: deprivation and rurality (two categories: rural and non-rural). Both ethnicity and rurality are measured in accordance with the categories used by the Office of National Statistics. ${ }^{35}$ Deprivation was measured using the index of multiple deprivation score (IMD) which includes various socioeconomic indicators at a local level. IMD score is matched (by Ipsos MORI) to the respondent via their postal code. ${ }^{33}$

\section{Practice characteristics}

Family practitioner practice-level data was obtained from the Health and Social Care Information Centre. Measures included: potential differences in the

\footnotetext{
iThe EuroQOL EQ 5D score is a measure of quality of life with an index value ranging from -1 to $1 .^{34}$ The EQ 5D 5L score is used in all waves of the study, except for the first wave (January-March 2012) which uses the EQ_5D_3L score.

ii Alzheimer's or dementia, angina or long-term heart problem, arthritis or long-term joint problem, asthma or long-term chest problem, blindness or severe visual impairment, cancer in the last five years, deafness or severe hearing impairment, diabetes, epilepsy, high blood pressure, kidney or liver disease, learning difficulty, long-term back problem, long-term mental health problem, long- term neurological problem, another long-term condition, and 'I would prefer not to say').
} 
availability of practitioner-the number of family practitioners per 1000 patients and the patient list size (divided by 1000), and factors that may impact on the patient's acceptability of seeking care-the percentage of family practitioners younger than 50 years, the percentage of family practitioners with a UK qualification, and the percentage of women family practitioners. Each observation at the practice level was recorded annually on 30 September of each respective year and, therefore, applied to two waves of the GPPS.

For further analysis, sexual orientation was interacted with the practice-level family practitioner age structure, gender composition and UK qualification structure. These interactions were included to investigate whether family practitioner characteristics differed in its effects on use by sexual orientation. For example, sexual orientation minorities may prefer to be seen by a practitioner of the same gender, age, or ethnicity; each of which may be due to misconceptions on the expectations and judgements of the patient to the provider (and vice versa). Each practice characteristic interaction was included in separate models; inclusion of all interactions would inflate the SEs (due to very small numbers). To assess whether there was inequity in use among sexual orientation groups for those with health problems we conducted additional analysis interacting each binary health condition with sexual orientation.

For all analyses, respondents with missing data on any variable, excluding sexual orientation, were not included for analysis. All analysis was performed with STATA 14 SE (Stata Corp. Stata Statistical Software: Release 14. 2015).

\section{Sensitivity analysis}

To test the robustness of our results, our analysis was replicated with sensitivity/robustness checks in three ways. First, we altered our family practitioner use variable to include an additional two model specifications, where visitation was within $0-6$ and $0-12$ months. This ensured the effect of sexual orientation on use was robust to the timing of the visit. Second, we recoded the family practitioner use variable to also include a nurse visit as some patients may visit a practice and not see a family practitioner. Finally, we tested whether non-response affected our results. By generating a binary variable for nonresponse and regressing this on family practitioner use and sexual orientation, it was possible to determine if non-response was correlated with sexual orientation.

\section{Patient involvement}

No patient involvement was included as part of this study.

\section{RESULTS}

There were 2807320 respondents over the six waves of the GPPS, 2740921 had a valid gender recorded (1 556909 women and 1184012 men). Non-response for patient characteristics among women reduced the sample to 1146024 (26.39\% reduction; 410885 fewer observations) and family practitioner practice characteristics to 1138653 (0.64\% reduction; 7370 fewer observations), respectively. Among men, non-response for the same factors reduced the sample to 923261 observations (22.02\% reduction; 260751 fewer observations), and family practitioner practice characteristics to 916643 observations $(0.72 \%$ reduction; 6618 fewer observations), respectively. The total sample loss due to nonresponse was 418255 (27.03\%) for women and 267369 $(22.74 \%)$ for men.

On average, $59.98 \%$ (59.57\% weighted) of women and $53.93 \%$ ( $48.03 \%$ weighted) of men visited a family practitioner within the past 3 months. Among the sample of women $91.87 \%$ (92.47\% weighted) identified as being heterosexual, $0.63 \%(0.77 \%$ weighted) lesbian, $0.44 \% \quad(0.63 \%$ weighted $)$ bisexual, $0.44 \% \quad(0.43 \%$ weighted) other, $3.83 \%$ (3.61\% weighted) 'prefer not to say' and $2.79 \%(2.09 \%$ weighted) did not answer. In the sample of men, $91.66 \%$ (91.41\% weighted) identified as being heterosexual, $1.68 \%$ (2.30\% weighted) gay, $0.48 \%$ $(0.58 \%$ weighted $)$ bisexual, $0.45 \% \quad(0.45 \%$ weighted $)$ other, $3.87 \%$ (3.84\% weighted) 'prefer not to say' and $1.86 \%$ (1.43\% weighted) did not answer. The full set of sample rates for each variable are provided in online supplementary table S1.

Regression results for family practitioner use in the past 3 months by sexual orientation are shown for women in table 1 (full regression output is available in online supplementary table S2). The ORs for lesbian women decreased, and for bisexual, other and 'prefer not to say' switched to lower use once patient characteristics were included (and was insignificant for nonrespondents). Adjusting for practice characteristics had little impact on the estimated ORs. For all minority sexual orientation groups, family practitioner use was lower in comparison with heterosexual women, this ranged from a $9 \%$ lower use for those preferring not to state their sexual orientation (OR $0.907,95 \%$ CI 0.881 to 0.935 ; absolute difference: -0.096 percentage points $95 \%$ CI -0.127 to -0.066$)$ to a $20 \%$ lower use for lesbian women (OR 0.803 , $95 \%$ CI 0.755 to 0.854 ; absolute difference: -0.220 percentage points $95 \%$ CI -0.281 to -0.158 ).

For men, only gay men and 'prefer not to say' were statistically significant from heterosexuals after adjusting for the patient and GP practice characteristics, shown in table 2 (full regression output is available in see online supplementary table S2). The ORs for gay men increased once patient characteristics were included, changed to reduced use for 'prefer not to say' respondents, and were insignificant for bisexual, other and non-respondents. Adjusting for practice characteristics had little impact on the estimated ORs. Family practitioner use was higher in comparison with heterosexual men for gay men (OR 1.218, 95\% CI 1.163 to 1.276; absolute difference: 1.967 percentage points $95 \%$ CI 
Table 1 Family practitioner visit in the past 3 months by sexual orientation among women $(N=1138653)$

\section{Unadjusted}

Adjusted for patient characteristics

\section{Adjusted for patient and} practice characteristics

\begin{tabular}{lllllllllll}
\hline \multicolumn{2}{l}{ Sexual orientation (base heterosexual) } & & & & & & & & \\
Lesbian & 0.912 & $(0.860$ to 0.966$)$ & $\mathrm{p}=0.002$ & 0.806 & $(0.758$ to 0.857$)$ & $\mathrm{p}<0.001$ & 0.803 & (0.755 to 0.854) & $\mathrm{p}<0.001$ \\
Bisexual & 1.245 & $(1.154$ to 1.343$)$ & $\mathrm{p}<0.001$ & 0.888 & $(0.818$ to 0.964$)$ & $\mathrm{p}=0.004$ & 0.887 & $(0.817$ to 0.963$)$ & $\mathrm{p}=0.004$ \\
Other & 1.163 & $(1.077$ to 1.257$)$ & $\mathrm{p}<0.001$ & 0.857 & $(0.788$ to 0.932$)$ & $\mathrm{p}<0.001$ & 0.857 & $(0.790$ to 0.935$)$ & $\mathrm{p}<0.001$ \\
$\begin{array}{l}\text { Prefer not to } \\
\text { say }\end{array}$ & 1.033 & $(1.006$ to 1.060$)$ & $\mathrm{p}=0.014$ & 0.908 & $(0.881$ to 0.936$)$ & $\mathrm{p}<0.001$ & 0.908 & $(0.881$ to 0.935$)$ & $\mathrm{p}<0.001$ \\
Not answered & 1.240 & $(1.204$ to 1.277$)$ & $\mathrm{p}<0.001$ & 1.015 & $(0.983$ to 1.048$)$ & $\mathrm{p}=0.356$ & 1.016 & (0.985 to 1.049) & $\mathrm{p}=0.315$ \\
\hline
\end{tabular}

Logistic regression of family practitioner use in the past 3 months.

SEs are robust to heteroskedasticity and clustered at the practice level.

Estimates are OR relative to the base of heterosexual.

$95 \%$ Cls in brackets.

0.151 to 0.243 ), and lower for those preferring not to state their sexual orientation (OR $0.957,95 \%$ CI 0.924 to 0.990 ; absolute difference: -0.044 percentage points $95 \%$ CI -0.079 to -0.010 ).

In additional analyses, we interacted sexual orientation with practice characteristics to test whether differences in family practitioner use by sexual orientation was driven by specific practice characteristics. Tables 3 and 4 give the estimated difference in the effects of three practice characteristics by sexual orientation for women and men, respectively (full output is available in online supplementary table S3 and S4 for women and men, respectively). The effects of the proportion of practice practitioners aged under 50 years was not statistically different for lesbian women compared with heterosexuals $(\mathrm{OR}=1.019$, 95\% CI 0.810 to 1.283$)$, or bisexual women compared with heterosexuals (OR=1.002, 95\% CI 0.762 to 1.317 ). An insignificant difference was also found for gay men compared with heterosexuals $(\mathrm{OR}=1.142,95 \%$ CI 0.976 to 1.337), and bisexual men compared with heterosexuals $(\mathrm{OR}=1.168,95 \%$ CI 0.873 to 1.563$)$. No difference was found for the proportion of practice practitioners that are UK qualified for lesbian women $(\mathrm{OR}=0.934$, $95 \%$ CI 0.765 to 1.142$)$ and bisexual women $(\mathrm{OR}=1.223$, $95 \%$ CI 0.966 to 1.550$)$, or gay men $(\mathrm{OR}=0.979,95 \%$ CI
0.845 to 1.134$)$ and bisexual men $(\mathrm{OR}=0.918,95 \%$ CI 0.716 to 1.177$)$ in comparison with their heterosexual counterparts. The proportion of the respondents' practice that are women practitioners did not differ in the effects on visits between lesbian ( $\mathrm{OR}=1.019,95 \%$ CI 0.810 to 1.283$)$ or bisexual women $(\mathrm{OR}=1.328,95 \% \mathrm{CI}$ 0.964 to 1.830 ) compared with heterosexual women, or bisexual men compared with heterosexual men $(\mathrm{OR}=0.934,95 \%$ CI 0.661 to 1.318$)$. A significant effect was found between gay men and heterosexual men (OR=1.238, 95\% CI 1.041 to 1.472$)$.

The estimates provided in online supplementary tables S5 and S6 give the relative odds of use for the respective sexual orientation group with that health condition in comparison with heterosexuals with that health condition. For example, the estimate of 1.194 for gay men with diabetes is interpreted as gay men with diabetes were $19.4 \%$ more likely to have used a family practitioner within the past 3 months than heterosexual men with diabetes. Differences in use for gay men with longterm heart problems, diabetes, learning difficulties and long-term mental health problems exist; for bisexual men only those with diabetes had different use rates than heterosexual men. The only significant difference in use for lesbian women was found for those with

Table 2 Family practitioner visit in the past 3 months by sexual orientation among men ( $N=916643)$

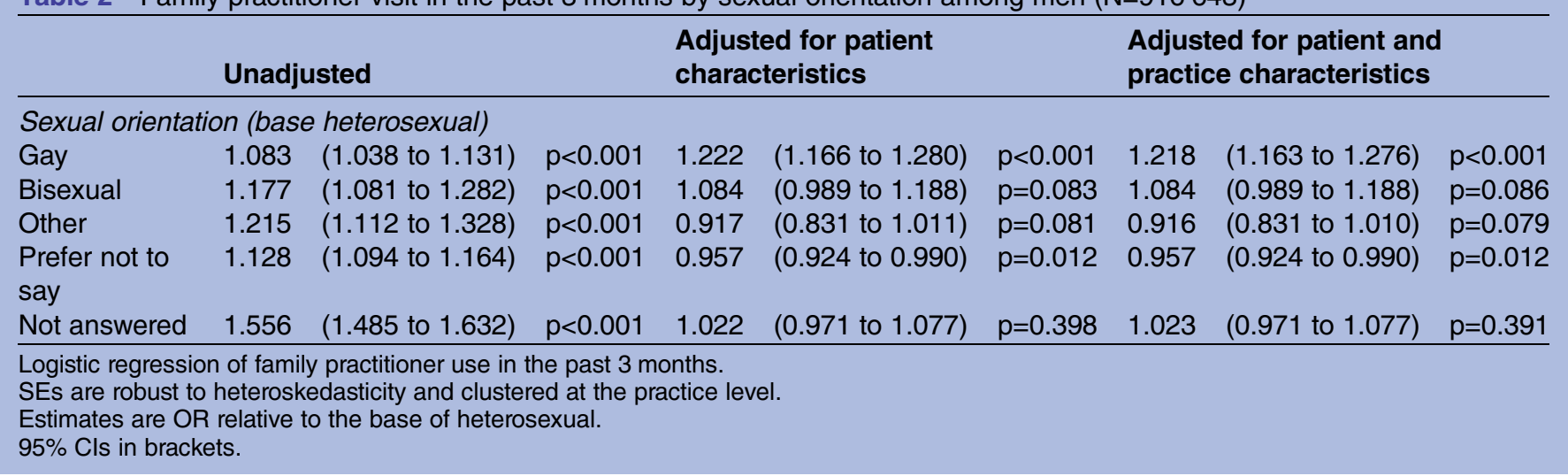


Table 3 Family practitioner visit in the past 3 months by sexual orientation among women ( $N=1138653)$ : practice characteristics interactions

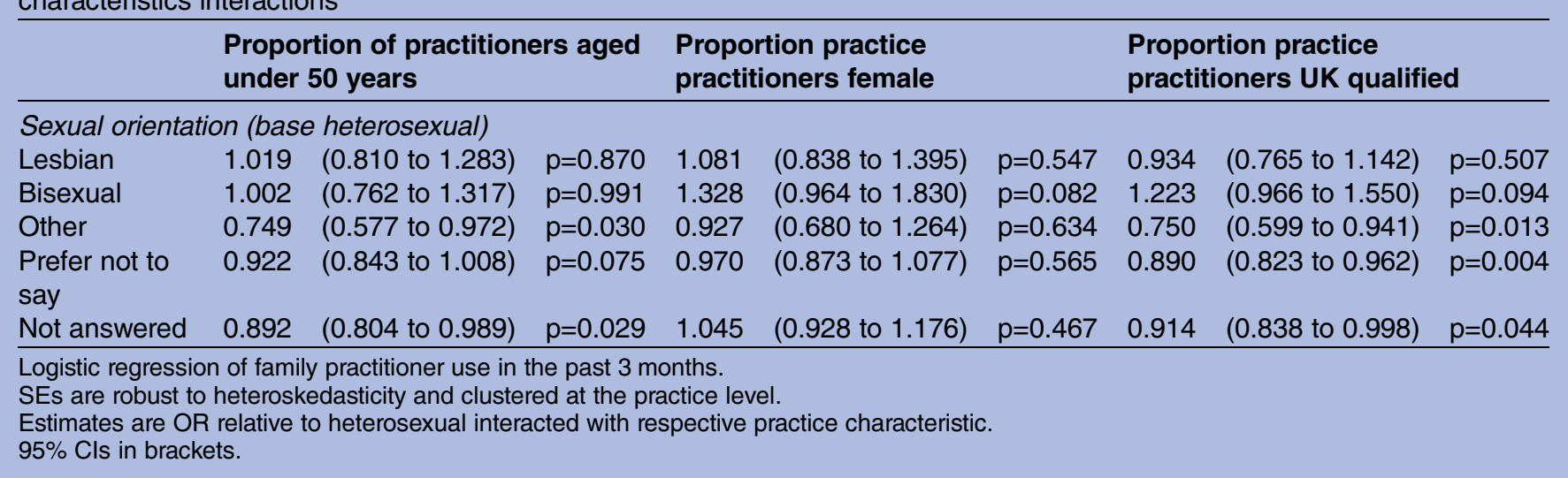

asthma or a long-standing health condition, whereas for bisexual women, differences exist for those with diabetes and a long-term mental health problem.

\section{Sensitivity analysis}

Several specification checks were conducted. First, we replicated the analysis varying the time interval for seeing a family practitioner from $0-6$ months to 0 12 months. For women, lesbians were less likely to have visited the family practitioner compared with heterosexual women across the alternate time intervals (see online supplementary table S7). For men, gay men were consistently more likely to have visited a family practitioner up to 12 months ago relative to their heterosexual counterparts (see online supplementary table S8). Bisexual men were consistently found to have no significant difference relative to their heterosexual counterparts. Second, we replaced family practitioner visits to (1) a nurse visit and (2) either a family practitioner or nurse visit. For women, lesbian women were less likely to visit a nurse, or either a nurse or practitioner within the past 3 months, than heterosexual women (see online supplementary table S9). Bisexual women were less likely to visit a nurse or practitioner, but no significant difference was found for nurse visits. For men, gay men were more likely to have seen a nurse, or either a nurse or practitioner, than heterosexual men in the past 3 months (see online supplementary table S10). A test of non-response found that sexual orientation and family practitioner use were correlated with nonresponse for men and women (see online supplementary table S11).

\section{DISCUSSION}

\section{Key results}

We sought to identify whether there are significant differences in family practitioner use by sexual orientation. Our first hypothesis was that the unconditional effects of sexual orientation on use are biased, partly reflecting the correlation between sexual orientation and need, availability and affordability of healthcare domains. We found the unconditional differences in lesbian women and gay men compared with their heterosexual counterparts were larger once adjustment was made for patient characteristics (age, ethnicity, work status, IMD score, religions status, rurality, EQ_5D score, acute ill health, medical condition, long-standing health condition and

Table 4 Family practitioner visit in the past 3 months by sexual orientation among men ( $N=916$ 643): practice characteristics interactions

\section{Proportion of practice practitioners aged under 50 years}

\section{Proportion of practice practitioners female}

\section{Proportion of practice practitioners UK qualified}

\section{Sexual orientation (base heterosexual)}

\begin{tabular}{|c|c|c|c|c|c|c|c|c|c|}
\hline Gay & 1.142 & (0.976 to 1.337 ) & $p=0.098$ & 1.238 & (1.041 to 1.472 ) & $p=0.016$ & 0.979 & (0.845 to1.134) & $p=0.779$ \\
\hline Bisexual & 1.168 & (0.873 to 1.563$)$ & $\mathrm{p}=0.295$ & 0.934 & (0.661 to 1.318 ) & $p=0.696$ & 0.918 & (0.716 to 1.177 ) & $p=0.500$ \\
\hline Other & 0.748 & (0.551 to 1.014 ) & $p=0.062$ & 0.947 & (0.673 to 1.331$)$ & $p=0.753$ & 0.780 & (0.609 to 0.998 ) & $p=0.048$ \\
\hline $\begin{array}{l}\text { Prefer not to } \\
\text { say }\end{array}$ & 1.008 & (0.909 to 1.119 ) & $p=0.875$ & 0.907 & (0.806 to 1.021 ) & $p=0.105$ & 0.885 & (0.809 to 0.968$)$ & $p=0.008$ \\
\hline Not answered & 0.882 & (0.751 to 1.035$)$ & $\mathrm{p}=0.123$ & 0.947 & (0.792 to 1.135$)$ & $p=0.556$ & 0.894 & (0.782 to 1.023 ) & $p=0.104$ \\
\hline
\end{tabular}


smoking status). Differences for bisexual women switched from greater odds to lower odds; and reduced in size for bisexual men. These suggest that the estimates of the effects of sexual orientation on use were confounded by patient characteristics. For example, reporting being a sexual orientation minority group may be negatively related with age, while younger women had higher rates of practitioner visits, and younger men had lower rates. Our second hypothesis was that having best accounted for confounding, significant differences exist in family practitioner use by sexual orientation status. This was motivated by the potential for patient acceptability to differ between sexual orientation groups based on preconceptions by (possibly) both patient and practitioner. We found significant differences in family practitioner use for lesbians compared with heterosexual women (OR $0.803,95 \%$ CI 0.755 to 0.854 ; absolute difference: -0.219 percentage points $95 \%$ CI -0.281 to $-0.157)$, and gay men compared with heterosexual men (OR 1.218, 95\% CI 1.163 to 1.276 ; absolute difference: 1.967 percentage points $95 \%$ CI 0.151 to 0.243$)$. Women who identified as bisexual, other, and 'prefer not to say', all had lower family practitioner use relative to heterosexual women, but greater use relative to lesbians.

To understand the role of the family practitioner in the effects of patient acceptability we interacted sexual orientation with several practice-level characteristics. Interaction models show that gay men were relatively more likely to visit a family practitioner than heterosexual men if their practice had a greater proportion of women family practitioners. The effects of an older or UK-trained practice did not differ by gay, lesbian or bisexual sexual orientation groups.

To test for differences in family practitioner use within health conditions we interacted each health condition by sexual orientation. Significant differences in use were found for gay men with long-term heart problems, diabetes, learning difficulties, and long-term mental health problem, for bisexual men with diabetes, for lesbian women with asthma or a long-standing health condition, and for bisexual women with diabetes compared with the respective heterosexual counterparts. While this may signify concerns of inequity in access for those with equal need, in the GPPS data it is not possible to determine whether these differences are due to differences in severity or patient compliance with long-term care.

\section{Strengths}

This study adds to the existing literature on family practitioner use by sexual orientation in two ways. First, the relatively large sample used allowed us to test sexual orientation differences while also accounting for potential confounding; thus permitting a clearer understanding of where policy may be best focused in order to reduce avoidable inequalities in health by sexual orientation. Second, the ability to match practice characteristics in the GPPS data enabled an investigation into the potential impacts of supply-side factors (gender, age and country of qualification) on patient acceptability of care, which could indicate potential barriers of access should misconceptions be evident between patient and practitioner.

\section{Limitations}

Our main outcome variable failed to account for the frequency and content of family practitioner visits. We were unable to ascertain the reason for the family practitioner visit, and as to whether the reason justified a visit. Our analysis was also limited to the use of family practitioners and did not provide any evidence of potential access issues to hospital or community-based care.

Until recently, few surveys had included sexual orientation as a question. Estimates on the total percentage of the population in England falling into each orientation are relatively unknown. Therefore, it is hard to know how representative our sample was of each sexual orientation. However, as the GPPS is taken from a random draw of the population of practices in England, this may not have been a concern unless minority sexual orientation groups were less likely to be registered with a practice. It is possible that minority sexual orientation groups did not respond to the survey, and this is not accounted for in the existing weights for representativeness supplied by the data holders.

The most recent Office of National Statistics (ONS) estimates indicated that among the male population of the UK, $1.1 \%$ identify as gay, $0.4 \%$ identify as bisexual; and among women, $0.6 \%$ identify as lesbian and $0.6 \%$ identify as bisexual. ${ }^{36}$ These estimates are likely to vary depending on which measurement is used to derive sexual orientation: identity, behaviour or attraction. The most common measurement is self-perceived identity used in this study and by the ONS, and a variety of other surveys. The sample sizes in this study are similar to ONS estimates with the exception of the gay men sample being larger in this study. Other studies that have used self-perceived identity contain a similar percentage of gay and lesbian individuals, and fall within roughly a one percentage difference of this study. ${ }^{6} 2226283036$ The possibility of respondents not reporting their true sexual orientation may be reduced as the GPPS is a postal survey where respondents do not have to tell an interviewer their sexual orientation face to face.

The variables used for adjustment are detailed, but further information would benefit the model. For example, IMD is only at the geographic level (lower super output area) not at the individual level; family practitioner practice variables are only available annually; and all health data were self-reported. In addition, the practice characteristics are imperfect proxies for the practitioner the patient sees, especially in large practices. Throughout it is assumed that all measures of the need for healthcare were captured, but unmet need may still exist should our measures of health not fully encapsulate the need for healthcare. Non-response is likely to bias 
our estimates, sexual orientation was found to be a significant predictor of non-response, however, the magnitude of the effect was small for gay and bisexual men as well as for lesbian and bisexual women.

Generalisability of the study results may be difficult due to differences in institutional settings, and potential different cultures and tolerance of minority sexual orientation groups.

\section{Comparisons with other studies}

Our main results for gay men complement findings in the $\mathrm{USA}^{22} 25$ and Europe ${ }^{24} \quad 27 \quad 28$ comparing male same-sex couples with males in heterosexual couples. This contrasts with other findings in the USA and Canada which provide evidence of no difference in practitioner use. ${ }^{623}{ }^{26}$ For lesbians, our results are similar to other studies from the USA and Canada, ${ }^{6} 202223$ several studies also find evidence that lesbian women are less likely to have had a health check-up (ie, a pap test or a cancer screening). ${ }^{6} 202125$ An Australian study found that lesbian women between the ages of 25 and 30 years were more likely to consult family practitioners contrasting with the findings of this study and the literature. ${ }^{7}$

A recent Stonewall study highlighted the poor perceptions of minority sexual orientation groups in the English healthcare system, and emphasised supply-side areas for improvement, such as provider training and awareness, as well as zero tolerance for instances of sexual orientation discrimination. ${ }^{11}$ The Stonewall findings are complemented by a study using the GPPS that found minority sexual orientation groups have worse healthcare experiences than heterosexuals. ${ }^{5}$ These findings suggest minority sexual orientation groups would have lower use relative to heterosexuals. While this may explain lower rates of use among lesbian women, our results for gay men contrast sharply, with our study finding relatively greater use for male minority sexual orientation groups.

\section{Implications}

Our findings show inequity in sexual orientation family practitioner usage. Having adjusted for several patient and practice factors that may impact on access to healthcare and confound the sexual orientation relationship, our results suggest more efforts could be made to overcome any barriers in the acceptability domain of healthcare access.

An acceptance of inequalities in use requires a value judgment on how inequitable use should be. Among women, it may be justifiable for lesbian and/or bisexual women to use family practitioners at a lower rate should we believe the difference is wholly explained by birth control and/or sexual reproductive services. Another study in Canada was able to account for birthing mothers and found it did not affect the results. ${ }^{6}$ Among men, it is not clear whether gay men and/or heterosexual men have sufficient family practitioner use. On the assumption that our model accounts for the relative need for healthcare, our results imply there is either significant unmet need for primary care among non-gay men, or significant overuse of primary care among gay men, with barriers to practitioner use seemingly occurring due to the gender distribution of practices. Further research aimed at evaluating the differences in family practitioner use is needed to understand whether the differences observed in this study are justified to the healthcare system.

\section{Twitter Follow William Whittaker at @HealthEcon_Will}

Contributors WW designed the study. SU drafted the initial manuscript and conducted the econometric analysis. All authors made substantial contributions to the study design and interpretation of the findings and contributed to the drafting of the article, gave final approval of the version to be published, and agree to be accountable for all aspects of the work in ensuring that questions related to the accuracy or integrity of any part of the article are appropriately investigated and resolved. SU is guarantor.

Funding This research received no specific grant from any funding agency in the public, commercial or not-for-profit sectors.

Competing interests All authors have completed the ICMJE uniform disclosure form and declare: no funding support for this work. WW reports grant funding for other work from the Department of Health Policy Research Programme and from NIHR and from NHS England for unrelated work. No other relationships or activities that could appear to have influenced the submitted work are declared. The Funders have not influenced the conduct of the study, nor the writing and submission of this paper.

Ethics approval Ethical approval was not required for this study. Ipsos MORI follow the Market Research Society Code of Conduct, notably, all personal data is destroyed upon survey completion and not transferred to the end user (the authors). Further, the GPPS is an assessment of service evaluation, precluding the necessity for medical research ethical approval.

Provenance and peer review Not commissioned; externally peer reviewed.

Data sharing statement No additional data are available.

Data access All authors had access to the data and can take responsibility for the integrity of the data and the accuracy of the data analysis.

Open Access This is an Open Access article distributed in accordance with the Creative Commons Attribution Non Commercial (CC BY-NC 4.0) license, which permits others to distribute, remix, adapt, build upon this work noncommercially, and license their derivative works on different terms, provided the original work is properly cited and the use is non-commercial. See: http:// creativecommons.org/licenses/by-nc/4.0/

\section{REFERENCES}

1. World Health Organization. Sexual health, human rights and the law. Geneva, Switzerland: WHO, 2015. http://apps.who.int/iris/bitstream/ 10665/175556/1/9789241564984_eng.pdf?ua=1 (accessed 9 Feb 2016).

2. Crombie IK, Irvine L, Elliott L, et al. Closing the health inequalities gap: an international perspective. World Health Organization, 2005 http://www.euro.who.int/_data/assets/pdf_file/0005/124529/E87934. pdf (accessed 9 Feb 2016).

3. Mayer $\mathrm{KH}$, Bradford JB, Makadon $\mathrm{HJ}$, et al. Sexual and gender minority health: what we know and what needs to be done. Am J Public Health 2008;98:989-95.

4. Bränström R, Hatzenbuehler ML, Pachankis JE. Sexual orientation disparities in physical health: age and gender effects in a population-based study. Soc Psychiatry Psychiatr Epidemiol 2016;51:289-301.

5. Elliott MN, Kanouse DE, Burkhart Q, et al. Sexual minorities in England have poorer health and worse health care experiences: a national survey. J Gen Intern Med 2015;30:9-16.

6. Tjepkema M. Health care use among gay, lesbian and bisexual Canadians. Health Rep 2008;19:53-64. 
7. McNair R, Szalacha LA, Hughes TL. Health status, health service use, and satisfaction according to sexual identity of young Australian women. Womens Health Issues 2011;21:40-7.

8. Conron KJ, Mimiaga MJ, Landers SJ. A population-based study of sexual orientation identity and gender differences in adult health. Am J Public Health 2010;100:1953-60.

9. McIntyre D, Thiede M, Birch S. Access as a policy-relevant concept in low- and middle-income countries. Health Econ Policy Law 2009;4:179-93.

10. Gulliford M, Figueroa-Munoz J, Morgan M, et al. What does 'access to health care' mean? J Health Serv Res Policy 2002;7: $186-8$.

11. Stonewall. Unhealthy attitudes, the treatment of LGBT individuals within health and social care services. Stonewall, 2014.

12. Council of Europe, Commissioner for Human Rights. Discrimination on grounds of sexual orientation and gender identity in Europe. Strasbourg: Council of Europe Publishing, 2011.

13. Wells and Polders. Gay and Lesbian people's experience of the health care sector in Guateng. 2012. http://www.out.org.za/index. php/library/reports\# (accessed 10 Feb 2016).

14. United Nations High Commissioner for Human Rights.

Discriminatory laws and practices and acts of violence against individuals based on their sexual orientation and gender identity. United Nations General Assembly, Human Rights Council 19th session, 2011. http://www.ohchr.org/Documents/lssues/ Discrimination/A.HRC.19.41_English.pdf (accessed 10 Feb 2016).

15. Dixon A, Le Grand J, Henderson J, et al. Is the NHS equitable?: a review of the evidence. LSE Health and Social Care, London School of Economics and Political Science, 2011. http://www.Ise.ac. uk/intranet/LSEServices/communications/pressAndInformationOffice/ PDF/DP11_2003 Is the NHS equitable.pdf (accessed 29 Jan 2016).

16. Goddard M, Smith P. Equity of access to health care services: theory and evidence from the UK. Soc Sci Med 2001;53:1149-62.

17. NHS England. Equality Analysis for 2014-15 and 2015-16 revenue allocations to Clinical Commissioning Groups and Area Teams. NHS England, 2014.

18. Department of Health. Resource allocation: weighted capitation formula. 7th edn. DoH, 2011.

19. Alencar Albuquerque G, de Lima Garcia C, da Silva Quirino G, et al. Access to health services by lesbian, gay, bisexual, and transgender persons: systematic literature review. BMC Int Health Hum Rights 2016;16:2.

20. Kerker BD, Mostashari F, Thorpe L. Health care access and utilization among women who have sex with women: sexual behavior and identity. J Urban Health 2006;83:970-9.

21. Diamant AL, Wold C, Spritzer K, et al. Health behaviors, health status, and access to and use of health care: a population-based study of lesbian, bisexual, and heterosexual women. Arch Fam Med 2000;9:1043-51.

22. Heck JE, Sell RL, Gorin SS. Health care access among individuals involved in same-sex relationships. Am J Public Health 2006;96:1111-18.
23. Strutz KL, Herring AH, Halpern CT. Health disparities among young adult sexual minorities in the U.S. Am J Prev Med 2015;48:76-88.

24. Wang J, Häusermann M, Vounatsou P, et al. Health status, behavior, and care utilization in the Geneva Gay Men's Health Survey. Prev Med 2007;44:70-5.

25. Buchmueller T, Carpenter CS. Disparities in health insurance coverage, access, and outcomes for individuals in same-sex versus different-sex relationships, 2000-2007. Am J Public Health 2010;100:489-95.

26. Wheldon CW. Are there differing patterns of health care access and utilization among male sexual minorities in the United States? J Gay Lesbian Soc Serv 2013;25:24-36.

27. King M, Nazareth I. The health of people classified as lesbian, gay and bisexual attending family practitioners in London: a controlled study. BMC Public Health 2006;6:127.

28. Bakker FC, Sandfort TGM, Vanwesenbeeck I, et al. Do homosexual persons use health care services more frequently than heterosexual persons: findings from a Dutch population survey. Soc Sci Med 2006;63:2022-30.

29. Chakraborty A, McManus S, Brugha TS, et al. Mental health of the non-heterosexual population of England. Br J Psychiatry 2011;198:143-8.

30. Everett BG, Mollborn S. Examining sexual orientation disparities in unmet medical needs among men and women. Popul Res Policy Rev 2014:33:553-77.

31. Ipsos MORI Social Research Institute. GP Patient Survey-technical annex. 2013-2014 annual report. London: Ipsos MORI, 2014. http:// gp-survey-production.s3.amazonaws.com/ archive/2014/July/ 1301375001_Technical20Annex 202013-2014_FINAL\%20v1.pdf (accessed 29 Jan 2016).

32. Nemes S, Jonasson JM, Genell A, et al. Bias in odds ratios by logistic regression modelling and sample size. BMC Med Res Methodol 2009:9:56.

33. Ipsos MORI Social Research Institute. GP Patient Survey-technical annex. 2011-2012 annual report. London: Ipsos MORI, 2012. http:// gp-survey-production.s3.amazonaws.com/ archive/2014/July/ 1301375001_Technical20Annex 202013-2014_FINAL\%20v1.pdf (accessed 29 Jan 2016).

34. Rabin $\mathrm{R}$, Oemar $\mathrm{M}$, Oppe $\mathrm{M}$, et al. EQ-5D-5L user guide: basic information on how to use the $E Q-5 D-5 L$ instrument. Rotterdam: EuroQol Group, 2011:22.

35. Office for National Statistics. Ethnicity and National Identity in England and Wales: 2011. Office for National Statistics, 2012. http:// www.ons.gov.uk/peoplepopulationandcommunity/culturalidentity/ ethnicity/articles/ethnicityandnationalidentityinenglandandwales/ 2012-12-11 (accessed 29 Jan 2016).

36. Joloza T, Evans J, O'Brien R, et al. Measuring sexual identity: an evaluation report. Newport: Office for National Statistics, 2010. http:// jeffdudgeon.com/download/Gay\%20rights/Gay\%20rights\% 20documents/Office\%20of\%20National\%20Statistics\%20(ONS)\% 20 Measuring $\% 20$ Sexual\%20Identity\%20report\%20September\% 202010.pdf (accessed 29 Jan 2016). 